\title{
The Level of Consumption Prediction and Analysis Based on the Grey Prediction Model
}

\author{
Xiaoge $\mathrm{Li}^{1, \mathrm{a}^{*}}$ \\ ${ }^{1}$ Pingdingshan Industrial College of Technology, Pingdingshan, Henan 467001, China \\ aemail:651953093@qq.com
}

Keywords: Grey Prediction Model; Household Consumption; Model Validation.

\begin{abstract}
With statistical forecast becoming more and more mature at home and abroad, the predicting theory and methodology has developed in depth to reach a relatively comprehensive stage. In this paper, a grey prediction model is established and applied in the analysis and prediction of China's household consumption. As the calculation result of the evaluation instance in this paper indicates, the model proposed is applicable for the analysis and prediction of household consumption with a relatively high accuracy.
\end{abstract}

\section{Introduction}

The household consumption is a form of monetary measurement which is a significant indicator that reflects the living standard as well as the material and cultural needs of people. It is also the quantized representation of total consumption of all the residents. Economic growth is mainly promoted by consumption demand, investment demand and export demand. Among the three factors, consumption demand has a long-lasting impact on economic growth. Under circumstance of global economy growth slowing down, expanding domestic demand and increasing household consumption has become the primary force to promote economic growth. Among the numerous factors that affect GDP, consumption contributes to GDP the most. It can be inferred from historical data that economic growth in our country would be greatly restrained once the contribution by consumption to GDP declines to be less than $50 \%$. Therefore, consumption must be highly valued and promoted to develop the economy.

Studying the existed theory and methodology and creatively applying them into social practice is the basic idea of this paper. Grey prediction[1] is the predicting process for system which contains both certain and uncertain information. It is usually carried out following the steps below. Firstly, identify the dissimilarity among the system factors and conduct cor-relativity analysis, generating a regular data sequence. Secondly, establish the differential equation model. Thirdly, validate the model. Finally, if the model passes the validation then it can be used for prediction. The grey prediction has the following advantages: easy to use, generally applicable and highly accurate.

\section{The establishment of the $G M(1,1)$ model}

To avoid the stochastic characteristics of the original sequence and provide useful information for the model, the original sequence is usually processed before establishing the grey prediction model. The sequence is generally processed by conducting a continuous sum calculation[2].

Assume that there are $n$ points in the sequence $X^{(0)}$ :

$$
X^{(0)}=\left\{X^{(0)}(1), X^{(0)}(2), \mathrm{L}, X^{(0)}(n)\right\}
$$

A new sequence is generated by conducting a continuous sum calculation

$$
X^{(1)}=\left\{X^{(1)}(1), X^{(1)}(2), \mathrm{L}, X^{(1)}(n)\right\}
$$

The differential equation for model $G M(1,1)$ is then derived as: 


$$
\frac{d X^{(1)}}{d X}+a X^{(1)}=\mu
$$

Where $a$ is the grey figure and $\mu$ is the grey action.

Assume that $\alpha$ is a parameter vector whose value is to be assigned,

$$
\alpha=\left(\begin{array}{l}
a \\
\mu
\end{array}\right)
$$

By applying the least square method, it can be derived that:

$$
\alpha=\left(B^{T} B\right)^{-1} B^{T} Y n
$$

Where

$$
B=\left(\begin{array}{rr}
-\frac{1}{2}\left[X^{(1)}(1)+X^{(1)}(2)\right] & 1 \\
-\frac{1}{2}\left[X^{(1)}(2)+X^{(1)}(3)\right] & 1 \\
M ~ M \\
-\frac{1}{2}\left[X^{(1)}(n-1)+X^{(1)}(n)\right] & 1
\end{array}\right), \quad Y_{n}=\left(\begin{array}{r}
X^{(0)}(2) \\
X^{(0)}(3) \\
M \\
X^{(0)}(n)
\end{array}\right)
$$

By solving the equation, the prediction model can be written as:

$$
\hat{X}^{(1)}(k+1)=\left[X^{(0)}(1) \frac{\mu}{a}\right] e^{-a k}+\frac{\mu}{a}, k=0,1, \mathrm{~L}, n
$$

Calculate $\hat{X}^{(1)}(i)$ basing on the prediction model. By conducting a continuous minus calculation for $\hat{X}^{(1)}(i)$, the predicted sequence $\hat{X}^{(0)}(i)$ is derived.

\section{Model validation}

It is only half done after the predicted sequence being calculated. What is of the same importance is the validation of the prediction model. Common methods used to validate the model are listed as follows[3,4]:

(1) Prior validation

Conduct a feasibility validation for model $G M(1,1)$ using the original sequence $X^{(0)}$. If the values of step-wise ratio $\sigma^{(0)}(k)=\frac{X^{(0)}(k-1)}{X^{(0)}(k)}$ all lie in the admissible region of $\left(e^{-2}, e^{2}\right)$, then $X^{(0)}$ is regarded to be smooth and the prediction model can be used for prediction.

(2) Residual error validation

(3) Cor-relativity validation

The correlation coefficient is defined as:

$$
\eta(k)=\frac{\min \min |\hat{X}(k)-X(k)+\rho \max \max | \hat{X}(k)-X(k)||}{|\hat{X}(k)-X(k)|+\rho|\hat{X}(k)-X(k)|}
$$

Where $\rho$ is called the resolution and $0<\rho<1$. Generally $\rho=0.5$.

The cor-relativity is defined as:

$$
r=\frac{1}{n} \sum_{k=1}^{n} \eta(k)
$$

Based on the formula above, firstly calculate the correlation coefficient between $\hat{X}^{(0)}(i)$ and original sequence $X^{(0)}(i)$, then calculate the cor-relativity. Determined by experience, if the cor-relativity is larger than 0.6 when $\rho=0.5$, the prediction model is considered as satisfying.

(4) Post validation

The Standard Deviation (SD) of original sequence: 


$$
S_{1}=\sqrt{\frac{\sum\left[X(0)-X_{t}^{(0)}\right]^{2}}{n-1}}
$$

The Standard Deviation (SD) of absolute error sequence:

$$
S_{2}=\sqrt{\frac{\sum\left[\Delta(0)-\Delta_{t}^{(0)}\right]^{2}}{n-1}}
$$

The variance ratio:

$$
C=\frac{S_{2}}{S_{1}}=\sqrt{\frac{\sum\left[\Delta(0)-\Delta_{t}^{(0)}\right]^{2}}{\sum\left[X(0)-X_{t}^{(0)}\right]^{2}}}
$$

The evaluation result for the prediction model corresponding to $\mathrm{C}$ : $\mathrm{c}<0.35$ GOOD; $\mathrm{C}<0.65$ QUALIFIED; $\mathrm{C} \geq 0.65$ UNQUALIFIED. If the prediction model passes residual error validation, cor-relativity validation and post validation, then it can be used for prediction.

\section{The simulation of the example}

By looking up in China Statistical Yearbook, the household consumption (hundred yuan) during 2010-2013 can be found, as the following table 1.

Table 1.The household consumption (hundred yuan) during 2008-2013

\begin{tabular}{|c|c|c|c|c|c|c|}
\hline Year & 2010 & 2011 & 2012 & 2013 & 2014 & 2015 \\
\hline Consumption & 81.81 & 91.03 & 106.10 & 118.21 & 130.12 & 145.60 \\
\hline
\end{tabular}

The original sequence is accordingly written as:

$$
X^{(0)}=\{81.81,91.03,106.10,118.21,130.12,145.60\}
$$

Considering sequence $X^{(0)}$, grey prediction is applicable as the values of step-wise ratio all lie in the interval $\left(e^{-2}, e^{2}\right)$.

Generate the new sequence by conducting a continuous sum calculation:

$$
X^{(1)}=\{81.81,171.84,277.94,396.15,526.27,671.87\}
$$

Calculate matrix B and the prediction model:

$$
\begin{array}{rlrl}
-\frac{1}{2}\left[X^{(1)}(1)+X^{(1)}(2)\right] & =-126.825, & -\frac{1}{2}\left[X^{(1)}(2)+X^{(1)}(3)\right]=-224.89 \\
-\frac{1}{2}\left[X^{(1)}(3)+X^{(1)}(4)\right] & =-337.045 & -\frac{1}{2}\left[X^{(1)}(4)+X^{(1)}(5)\right]=-461.21 \\
-\frac{1}{2}\left[X^{(1)}(5)+X^{(1)}(6)\right] & =-599.07
\end{array}
$$

We obtain that

$$
B=\left(\begin{array}{rr}
-126.825 & 1 \\
-224.89 & 1 \\
-337.045 & 1 \\
-461.21 & 1 \\
-599.07 & 1
\end{array}\right), \quad Y_{n}=\left(\begin{array}{r}
91.03 \\
106.10 \\
118.21 \\
130.12 \\
145.60
\end{array}\right)
$$


By calculating $\left(B^{T} B\right)^{-1} B^{T} Y_{n}=\left(\begin{array}{c}-0.1432 \\ 40.1659\end{array}\right)$, we have that:

$$
\alpha=-0.1432, \mu=40.1659, \frac{\mu}{\alpha}=\frac{40.1659}{-0.1432}=-280.488
$$

The prediction model $G M(1,1)$ is derived as:

$$
\hat{X}^{(1)}(K+1)=321.480^{0.1432 k}-280.488 \text {. }
$$

According to the (7), we then have that the relative error is less than $2.5 \%$, so the model has a relatively high accuracy.

Combining to the (8)-(11), we obtain that $r=0.6011$, which meets the requirement that $r>0.6$ when $\rho=0.5$ and indicates that the prediction model has a relatively high accuracy. The value of $\mathrm{C}$ indicates that the prediction model is highly accurate and can be used for prediction.

Predict China's household consumption in the future three years, according to the formula:

$$
\hat{X}^{(1)}(K+1)=321.480^{0.1432 k}-280.488
$$

Conduct a continuous minus calculation:

$$
\begin{aligned}
& \text { 2016: } \hat{X}^{(0)}(7)=162.723 \\
& \text { 2017: } \hat{X}^{(0)}(8)=180.110 \\
& \text { 2018: } \hat{X}^{(0)}(9)=201.163
\end{aligned}
$$

\section{Conclusions}

As the data indicates, the household consumption has been growing steadily. However, the economic development goes against household consumption[5] to a certain extent by observing the data carefully. On one hand, people's income has been increasing continuously due to the steady economic growth. On the other hand, the consumption scale is only growing slowly and sometimes undergoes a negative growth. To turn around the economy has become an urgent matter of the moment for the government. Considering the generally growing trend of the economy, it can be inferred that the rapid growth would still last for a time, then the growth rate would undergo a gradually declining process but a steady growth is still likely to be maintained. What needs to be done is to overcome the current limitations, strive to adjust the structure of economy, actively promote the idea of environment protection and green growth, thoroughly implement scientific outlook on development and try the best to realize the healthy and rapid development of economy.

\section{References}

[1] G.X. Xu, Statistical forecasting and decision-making, Shanghai university of finance and economics press, Shanghai(2008).

[2] S.Y. Jia, X.Y. Ji, The mathematical model and application in the field of economic practice, Vol.5(2011), p.79-81.

[3] X.M. Wang, Dynamic customer requirements analysis based on the improved grey forecasting model, Systems Engineering - Theory\&Practice,Vol.30(2011), p.1380-1386.

[4] C.H. Chen, L.P. Khool, W. Yan, A strategy for acquiring customer requirement patterns using laddering technique and ARTZ neural network, Advanced Engineering Informatics, Vol.16(2002), No.3. p. 229-240.

[5] X.C. Xu, A study of the problem of national economic accounting and statistics of China, Peking University press, Beijing(2010). 